\title{
Sodium valproate for the treatment of mania in a patient with Charcot-Marie-Tooth disease
}

Charcot-Marie-Tooth disease (CMT), also known as Hereditary Sensory Motor Neuropathy (HSMN) is the most common inherited peripheral neuropathy and it can follow autosomal dominant, $\mathrm{X}$ linked, autosomal recessive and even a sporadic inheritance pattern. ${ }^{1}$ Very few studies have evaluated psychiatric problems in this disease ${ }^{2,3}$, but there is a 30\% lifetime prevalence of psychiatric disorder in patients with CMT. ${ }^{2}$ Depression and phobic disorder are commonly occurring psychiatric comorbidities. ${ }^{3}$ After obtaining informed consent, we report the case of a patient with Charcot-Marie-Tooth disease presenting with a manic episode and the treatment thereof with sodium valproate.

Mr. L, a 28 year old male, presented with a two month history of talkativeness, irritable mood, increased physical activity and disturbed sleep. At the time of presentation, he was noted to be singing religious songs and had grandiose plans for the future. There was an increased physical activity throughout the day without associated fatigue. At times, he would even dance and he frequently cracked jokes with other patients.

He had a history of several similar episodes in the past 10 years, almost one per year. In addition to this, he experienced generalized tonic-clonic seizures as a consequence of a head injury he sustained at the age of 14 years. He received antiepileptic medications (initially Phenytoin sodium and later Divalproex sodium) and remained seizure free for 8 years. There is also a history of progressive weakness and atrophy of his limbs, which had initially started in the distal, followed by the proximal aspects of both lower limbs, and later progressed to affect the upper limbs. As a result, he had difficulty in climbing stairs and getting up from a seated position. The patient's younger sister, who had a similar history of weakness, atrophy and deformity of her limbs, had suffered from several depressive episodes over a period of 8 years.

Investigations of patient i.e. haematology and biochemical tests were found to be normal. Magnetic resonance imaging of the patient's brain as well as visual evoked potential and brainstem auditory evoked response were normal. The nerve conduction studies showed decreased conduction velocity of $<20 \mathrm{~m} / \mathrm{s}$; with prolonged distal latencies, decreased compound muscle action amplitude and non-recordable sensory nerve axonal potential. Biopsy of the left sural nerve revealed "onion bulb" formation, fibrocystic proliferation, and increased endoneurial connective tissue. After psychiatric evaluation, he was diagnosed as a case of bipolar affective disorder, current episode mania with psychotic symptoms. The diagnosis of Charcot-Marie-Tooth disease was also entertained after consultation with a team of neurologists and psychiatrists. The patient was commenced on sodium valproate $1000 \mathrm{mg}$ per day for treatment of mania. Initially, risperidone $4 \mathrm{mg}$ was added to control aggression. Over a period of four weeks, his manic symptoms improved significantly and he achieved complete remission in approximately 10 weeks.

Charcot-Marie-Tooth disease is a chronic, disabling disorder and may be associated with psychiatric problems such as depression and anxiety as explained by the stress hypothesis. There is no evidence of an association between bipolar disorder and Charcot-Marie-Tooth disease and this is the first case report of such a rare combination.

Sodium valproate is a known mood stabilizing agent which has a neurotrophic effect in several regions of the cerebral cortex (dentate gyrus and hippocampus), mediated by the Extracellular signal Related Kinase (ERK) pathway. ${ }^{4}$ Valproate also causes proliferation and maturation of neuronal stem cells. ${ }^{4}$ Valproic acid is helpful in improving the formation of defective neurons seen in HSMN via the c-Jun N-terminal Kinase (JNK) signaling pathway. ${ }^{5}$ Since valproate plays a significant role in the repair of morphological defects of neurons through the ERK and JNK pathways in bipolar disorder and HSMN, its use will be of importance in the context of the two conditions co-existing. As there is no prescribed guideline for the management of this particular comorbidity, the role of valproic acid needs to be evaluated in future research.

\section{S Kumar Kar', AK Panda², A Kamboj', O Prakash' 'Department of Psychiatry, Institute of Human Behavior and Allied Sciences (IHBAS), Delhi, India \\ 2Department of Neurology, Institute of Human Behavior and Allied Sciences (IHBAS), Delhi,India}

\section{References}

1. Pareyson D, Marchesi C. Diagnosis, natural history, and management of Charcot-Marie-Tooth disease. Lancet Neurol 2009; 8(7):654-67.

2. Inczedy-Farkas G, Remenyi V, Gal A, Varga Z, Balla P, Udvardy-Meszaros A, Bereznai B, Molnar MJ. Psychiatric symptoms of patients with primary mitochondrial DNA disorders. Behav Brain Funct 2012 ; 13; 8:9.

3. Kalkman JS, Schillings ML, Zwarts MJ, van Engelen BG, Bleijenberg G. Psychiatric disorders appear equally in patients with myotonic dystrophy, facio-scapulo-humeral dystrophy, and hereditary motor and sensory neuropathy type I. Acta Neurol Scand 2007 ; 1 15(4):265-70.

4. Hao Y, Creson T, Zhang L, Li P, Du F, Yuan P, et al. Mood Stabilizer Valproate Promotes ERK Pathway-Dependent Cortical Neuronal Growth and Neurogenesis. The Journal of Neuroscience 2004; 24(29): 65906599.

5. Yamauchi J, Torii T, Kusakawa S, Sanbe A, Nakamura K, Takashima S, et al. The mood stabilizer valproic acid improves defective neurite formation caused by Charcot-Marie-Tooth disease-associated mutant Rab7 through the JNK signaling pathway.J Neurosci Res 2010 ; 88(14):3189-97. 